\title{
Family functioning at meals relates to adherence in young children with type 1 diabetes
}

\author{
Susana R Patton, ${ }^{1}$ Carrie Piazza-Waggoner, ${ }^{2}$ Avani C Modi, ${ }^{2}$ Lawrence M Dolan ${ }^{3}$ and Scott W Powers ${ }^{2}$ \\ 'Division of Child Behavioral Health, Department of Pediatrics and Communicable Diseases, C.S. Mott Children's Hospital and the University of Michigan, Ann \\ Arbor, Michigan, Divisions of ${ }^{2}$ Behavioral Medicine and Clinical Psychology and ${ }^{3}$ Endocrinology, Cincinnati Children's Hospital Medical Center, Department of \\ Pediatrics, University of Cincinnati College of Medicine, Cincinnati, Ohio, USA
}

\begin{abstract}
Aims: This study examined associations between mealtime family functioning, dietary adherence and glycaemic control in young children with type 1 diabetes mellitus (T1DM). We hypothesised that poorer family functioning would correlate with poorer dietary adherence and glycaemic control.

Methods: Thirty-five families of children ( $M=5.6 \pm 1.5$ years) with T1DM had meals videotaped in their home, which were coded for family functioning according to the McMaster Interaction Coding System. Children's dietary adherence was assessed according to deviations from the prescribed number of carbohydrate units per meal and recommended carbohydrate intake levels per day. Glycaemic control was measured via 14 days of self-monitoring of blood glucose levels.

Results: Findings demonstrated significant negative associations between children's dietary adherence and two dimensions of family functioning: Task Accomplishment ( $r=-0.43, P=0.03)$ and Behavioral Control $(r=-0.54, P=0.00)$. Affect Management correlated negatively with the percent of blood glucose levels below the normal range $(r=-0.33, P=0.05)$. Eleven families $(31 \%)$ of young children with type 1 diabetes demonstrated mealtime family functioning in the unhealthy range.

Conclusions: This was the first study to examine the relationship between mealtime family functioning and children's dietary adherence and glycaemic control in families of young children with T1DM. Previous research has found mealtime family functioning to be impaired in families of young children with T1DM when compared with families of children without diabetes. Research is needed to determine if family functioning and dietary adherence can be improved via specific family-based behavioural training around mealtimes.
\end{abstract}

Key words: adherence; diet; type 1 diabetes mellitus; youth.

Type 1 diabetes mellitus (T1DM) is a common paediatric chronic illness that impairs glucose functioning and puts patients at increased risk for blood glucose levels that are outside of the

\section{Key Points \\ 1 Families of young children with type 1 diabetes mellitus (T1DM) have poorer family functioning at mealtimes when compared with families of same-aged children without diabetes. \\ 2 Poorer family functioning at mealtimes is correlated with poorer dietary adherence in families of young children with T1DM. In contrast, parents with effective emotional expression at mealtimes have children with fewer low blood glucose concentrations \\ 3 Interventions to improve diabetes adherence and health out- comes for young children with T1DM should include behav- ioural components that target improved family functioning at mealtimes.}

Correspondence: Dr Susana R Patton, Division of Child Behavioral Health, University of Michigan, 1924 Taubman Centre, Box 0318, 1500 E. Medical Center Dr, Ann Arbor, MI 48109-0318, USA. Fax: +1 734936 6897; email: susanap@med.umich.edu

Accepted for publication 13 May 2009 normal range. ${ }^{1}$ Research in children with T1DM has focused on understanding factors that lead to better adherence to self-care and improved glycaemic control. One factor which has been the subject of many studies is the role of family functioning in the management of T1DM. ${ }^{2-6}$ It is known that family functioning factors, including family conflict, ${ }^{3}$ lack of responsibility taking for diabetes care tasks ${ }^{4}$ and perceptions of non-supportive parental behaviours ${ }^{5}$ are related to poorer diabetes adherence and glycaemic control in children with T1DM. In contrast, research has found a relationship between better diabetes adherence and families' guidance/control, a generally positive dimension of family functioning. ${ }^{6,7}$

An emerging area of research in T1DM is the role of family functioning within the context of mealtimes. Mealtimes provide the optimal context to examine adherence in TIDM because they involve multiple components of diabetes self-care, including blood glucose testing, insulin administration and dietary planning to ensure children consume an adequate amount of carbohydrates to match insulin levels. ${ }^{1}$ Moreover, for parents of young children, mealtimes are universally challenging because of problems with food refusal, picky eating, disruptions at mealtime behaviours and non-compliance with parental requests. ${ }^{8-10}$ One observational study, which compared family functioning in families of young children with TIDM and matched controls, 
found significantly poorer functioning for families of young children with TIDM, suggesting that the rigours of following a diabetes treatment plan may increase the risk of unhealthy functioning at mealtimes for families of young children with TIDM. ${ }^{11}$ What is not known is if the differences observed in family functioning at mealtimes also translate into greater diabetes risk for children. Thus, the goal of the current study was to extend the mealtime research by examining the impact of family functioning on dietary adherence and glycaemic control in families of young children with T1DM. We hypothesised that poorer family functioning, as evidenced by lower scores on the Mealtime Interaction Coding System (MICS), would be related to poorer dietary adherence and average daily glycaemic control for families of young children with TIDM.

\section{Research Design and Methods}

\section{Participants}

Thirty-five children with T1DM and their families participated in the current study. Families were recruited from the Pediatric Diabetes Center (PDC) at a tertiary hospital in the mid-western region of the United States. Eligibility criteria were: (i) a child between 2 and 8 years old; (ii) a confirmed diagnosis of T1DM for at least 1 year; (iii) no other diseases/conditions known to affect growth; and (iv) English spoken in the home. Within the PDC, 83 families were contacted to participate in clinical research. The first 35 families who agreed to complete both questionnaires and videotaping of home meals were enrolled in the current study.

Children who participated followed all types of insulin management. However, the majority (77\%) followed a conventional insulin regimen which consisted of two injections each day of a short and intermediate-acting insulin (e.g. Humalog/NPH) combined with one daily injection of a long-acting insulin (e.g. Lantus). The remaining eight children followed an intensive insulin regimen consisting of four to five insulin injections (e.g. Novolog and Lantus) or a continuous insulin infusion pump. At time of recruitment, $75 \%$ of young children with TIDM were using conventional insulin therapy.

\section{Procedure}

This study received Institutional Review Board approval prior to participant recruitment. Families were contacted about the study via a letter from the Principal Investigator and a follow-up telephone call made by a member of the research team. Families who consented to participate scheduled a home visit with a research assistant to complete the informed consent and begin the study. Data concerning children's dietary intake and family functioning at mealtimes were collected from three representative home meals which were videotaped according to a standardised protocol. ${ }^{9,10}$ Videotaped meals included lunches or dinners only. After completion of the meal, parents were asked to rate the typicality of the meal based on the target child's behaviour, food intake and the mealtime interactions of the family on a scale of 1 (not at all typical) to 5 (most typical possible). ${ }^{10}$ Only meals with ratings of 3 (typical) and higher were included. The mean number of home visits needed to obtain at least three representative meals was $3.11 \pm 0.33$. At study completion, families were reimbursed $\$ 70$ for their time and effort.

\section{Measures \\ Dietary adherence}

Children's diet records from each of the videotaped meals were analysed by a registered dietitian using the Minnesota Nutrition Data System (Nutrition Coordinating Center, University of Minnesota, 1996). For children on conventional therapy $(n=27)$, we calculated two measures of dietary adherence based on the number of carbohydrate units added or deleted from each videotaped meal that deviated from the child's individual diet plan for that meal. ${ }^{12}$ For this measure, a carbohydrate unit was equal to $15 \mathrm{~g}$ of carbohydrates. If the child ate more than the number of carbohydrate units recommended for the specific meal, the number of units in excess were counted as 'units added'. Similarly, if the child ate less than the number of carbohydrate units recommended, the number of units not eaten was counted as 'units deleted'. Exceptions to this measure occurred if the family indicated they were purposely deviating from the recommended carbohydrate intake to offset planned vigorous activity (e.g. carbohydrate loading) or to treat a low blood glucose value. ${ }^{12,13}$ These measures were also not used in the eight children who followed an intensive insulin regimen, because we did not have a record of children's pre-meal insulin doses and individual insulin to carbohydrate ratios. To calculate the deviation scores, children's meal records were reviewed by a team of trained research assistants. Inter-rater reliability was assessed using a Kappa coefficient. The Kappas were 0.88 for additions and 0.81 for deletions, which exceed the minimum considered acceptable for inter-rater reliability. ${ }^{14}$

\section{Children's daily glycaemic control}

To measure children's daily glycaemic control during the videotaping, families used a standardised FreeStyle (TheraSense, Alameda, CA, USA) home blood glucose meter. Families were instructed to use this metre to test their child's blood glucose at least four times daily for the 2 weeks they were also completing the mealtime videotaping. On average, children had $76 \pm 32$ blood glucose readings (range 31-159) or approximately $4.8 \pm$ 1.8 blood glucose checks per day of recording.

\section{Family functioning}

Family functioning at mealtimes was assessed using the MICS, an observational coding system based on the McMaster Model of Family Functioning. ${ }^{15}$ The MICS is a reliable and valid measure of family functioning which has been used previously in families of chronically and non-chronically ill children. ${ }^{16,17}$ The MICS evaluates family functioning according to six dimensions (task accomplishment, communication, affect management, interpersonal involvement, behavioural control, roles) and one separate scale of general functioning. For each of the dimensions, a 7-point scale is used to indicate quality of family functioning according to the following scale: 1 = very unhealthy and $7=$ very healthy). A clinical cut-off score can be applied to 
each dimension, with scores of 5 and higher suggesting functioning in the healthy range. Per MICS protocol, coding began with the start of the meal, which may be indicated by the presentation of food and the initiation of eating by at least one family member. Coding concluded at the end of the meal or after $20 \mathrm{~min}$, whichever occurred first. Two psychology postdoctoral fellows completed training with the developers of the MICS and for this study completed coding and reliability assessment. Coders for this study were blind to children's disease status. Reliability was evaluated based on 33\% of the meals and inter-rater reliability (intraclass correlation coefficient) for each dimension of the MICS ranged from $r=0.66-0.93$, suggesting good reliability. ${ }^{18}$

\section{Data analyses}

All data were analysed using SPSS statistical software. ${ }^{19}$ Descriptive data were calculated for sample characteristics and variables of interest (e.g. MICS scores, children's dietary adherence). To examine children's average glycaemic control during the study, we used the percent of blood glucose tests that fell above, below and within the target range for blood glucose control for young children (e.g. 3.8-10 mmol/L). Pearson product-moment correlations were used to examine associations between MICS scales, dietary adherence and daily glycaemic control.

\section{Results \\ Participants}

Table 1 presents descriptive statistics for the 35 children with T1DM who participated in this study as well as mean scores for each dimension of the MICS and mean scores for dietary adherence. With respect to family functioning, 11 families demonstrated scores on the individual dimensions of the MICS that were in the unhealthy range based on established clinical cutoffs (e.g. scores $<5 ;^{15}$ ).

\section{Family functioning and health outcomes}

Table 2 lists the correlations between each dimension of the MICS and children's dietary adherence and average glycaemic control. We predicted that families with poorer family functioning at mealtimes would have children with poorer dietary adherence and more blood glucose checks outside of the normal range. Consistent with our hypothesis, we found significant negative correlations between two dimensions of family functioning, Task Accomplishment and Behavioural Control, and the number of carbohydrate units children deleted from the videotape meals ( $r=-0.43$ and $r=-0.54$, respectively). A statistical trend was also found for poorer Role Functioning and the number of carbohydrate units deleted $(r=-0.36, P=0.07)$. With respect to carbohydrate additions, no significant relationships were found with MICS scales. When we correlated the dimensions of the MICS with the percent of readings below the normal range, we found a negative relationship with families' Affect Management $(r=-0.33, P=0.05)$, suggesting that families who demonstrate appropriate emotional expression during the meal have children who experience fewer low blood glucose

\begin{tabular}{|c|c|c|}
\hline Variable & M & SD \\
\hline Age (years) & 5.6 & 1.6 \\
\hline Weight (kg) & 22.3 & 5.0 \\
\hline Height (cm) & 113.2 & 11.6 \\
\hline Blood glucose level (mmol/L) & 11.8 & 2.7 \\
\hline $\mathrm{HbA1c}$ & 8.3 & 1.0 \\
\hline Daily carbohydrate intake & 186 & 54 \\
\hline \multicolumn{3}{|l|}{ Dietary deviation scores† } \\
\hline Carbohydrate unit additions (per meal & 0.20 & 0.30 \\
\hline Carbohydrate unit deletions (per meal & 0.37 & 0.74 \\
\hline \multicolumn{3}{|l|}{ MICS dimensions $\ddagger$} \\
\hline Task Accomplishment & 4.64 & 1.41 \\
\hline Communication & 4.56 & 1.00 \\
\hline Affect Management & 4.60 & 1.08 \\
\hline Interpersonal Involvement & 4.56 & 1.32 \\
\hline Behavioural Control & 4.92 & 1.19 \\
\hline Roles & 4.52 & 1.19 \\
\hline \multirow[t]{2}{*}{ Overall Family Functioning } & 4.56 & 1.19 \\
\hline & Frequency & $\%$ \\
\hline Gender (Boy) & 14 & 40 \\
\hline \multicolumn{3}{|l|}{ Race } \\
\hline White & 29 & 83 \\
\hline Non-White & 6 & 17 \\
\hline \multicolumn{3}{|l|}{ Socioeconomic status§ } \\
\hline 1 & 6 & 17 \\
\hline$\|$ & 0 & 0 \\
\hline III & 8 & 23 \\
\hline IV & 16 & 46 \\
\hline V & 5 & 14 \\
\hline \multicolumn{3}{|l|}{ Marital status } \\
\hline Married & 28 & 80 \\
\hline
\end{tabular}

†Average of deviation in carbohydrate units for all three meals. Higher scores reflect poorer dietary adherence. $¥$ Mealtime Interaction Coding System (MICS). Scores range from 1 to 7, with higher scores indicating better functioning. §The Hollingshead Four-Factor Scale is measured from I (lowest level) to V (highest level).

concentrations. Likewise, we found a statistical trend between families' Affect Management and the percent of readings above the normal range for children $(r=0.31, P=0.07)$, suggesting that families with better ability to manage emotions at mealtimes have children with higher glycaemic concentrations. Contrary to our hypothesis, no other dimensions of the MICS were found to correlate with children's glycaemic control.

\section{Discussion}

This is the first study to examine the relationship between family functioning at mealtimes and children's dietary adherence and average glycaemic control. The results are consistent with previous research examining mealtimes in families of young children with TIDM. Specifically, the extant literature demonstrates negative correlations between children's 
Table 2 Correlations between family functioning and health outcome variables

\begin{tabular}{|c|c|c|c|c|c|c|c|c|c|c|c|c|c|}
\hline Variables & 1 & 2 & 3 & 4 & 5 & 6 & 7 & 8 & 9 & 10 & 11 & 12 & 13 \\
\hline $\begin{array}{l}\text { Average daily blood } \\
\text { glucose }\end{array}$ & 1 & $-0.93^{\star \star}$ & $-0.57 * \star$ & $0.96 * *$ & -0.11 & -0.003 & 0.27 & -0.32 & -0.17 & -0.15 & -0.06 & 0.57 & -0.11 \\
\hline$\%$ values in range & - & 1 & $0.35^{\star}$ & $-0.96 * \star$ & 0.02 & 0.02 & -0.27 & 0.03 & 0.09 & 0.08 & 0.02 & $-0.39 *$ & -0.27 \\
\hline$\%$ values below & - & - & 1 & $-0.55^{\star *}$ & -0.09 & -0.15 & $-0.33^{\star}$ & -0.08 & -0.05 & 0.01 & -0.009 & -0.16 & 0.09 \\
\hline$\%$ values above & - & - & - & 1 & -0.005 & 0.01 & 0.31 & -0.006 & -0.07 & -0.08 & -0.02 & $0.38^{*}$ & -0.03 \\
\hline Task Accomplishment & - & - & - & - & 1 & $0.70 * \star$ & $0.66^{* *}$ & $0.67 * \star$ & $0.75^{\star *}$ & $0.75^{\star *}$ & $0.82^{\star \star}$ & $-0.43^{\star}$ & 0.05 \\
\hline Communication & - & - & - & - & - & 1 & $0.75^{\star \star}$ & $0.83^{* *}$ & $0.69 * *$ & $0.72^{\star *}$ & $0.88^{* *}$ & -0.22 & -0.05 \\
\hline Affect Management & - & - & - & - & - & - & 1 & $0.70 * *$ & $0.70^{\star *}$ & $0.68^{* *}$ & $0.82^{\star *}$ & -0.17 & 0.16 \\
\hline Interpersonal Involvement & - & - & - & - & - & - & - & 1 & $0.72^{\star \star}$ & $0.68^{\star *}$ & $0.86 * *$ & -0.18 & 0.006 \\
\hline Behavioral Control & - & - & - & - & - & - & - & - & 1 & $0.72^{\star \star}$ & $0.80 * *$ & $-0.54 * \star$ & 0.13 \\
\hline Roles & - & - & - & - & - & - & - & - & - & 1 & $0.79 * \star$ & -0.36 & 0.35 \\
\hline Overall Family Functioning & - & - & - & - & - & - & - & - & - & - & 1 & -0.25 & -0.02 \\
\hline $\begin{array}{l}\text { \# carbohydrate units } \\
\text { deleted from meals }\end{array}$ & - & - & - & - & - & - & - & - & - & - & - & 1 & -0.32 \\
\hline $\begin{array}{l}\text { \# carbohydrate units } \\
\text { added to meals }\end{array}$ & - & - & - & - & - & - & - & - & - & - & - & - & 1 \\
\hline
\end{tabular}

disruptive behaviour at mealtimes and the number of carbohydrate units deleted from children's meals. Similarly, the literature demonstrates negative correlations between parents' use of coercive management strategies and the number of carbohydrate units deleted from children's meals. ${ }^{20}$ Within the MICS, Task Accomplishment reflects the flow of the meal and how well families are able to minimise or respond to disruptions to the meal (e.g. complaints about the meal, members not eating, members leaving the table before the end of the meal). The Behavioural Control domain reflects parents' management style for the meal and if they demonstrate a style that is flexible and responsive to the child's behaviour, a style that is disorganised and chaotic, or a style that is overly rigid. ${ }^{15,21}$ Thus, common to the literature, the results of this study suggest that children with TIDM may experience poorer dietary adherence if they experience family meals characterised by frequent disruptions and their parents use primarily rigid and coercive management strategies during the meal.

This study has implications for research and clinical care in families of young children with T1DM. From a research perspective, this study supports the existing literature linking diabetes adherence with better family functioning. ${ }^{6,7}$ Moreover, our use of an observational measure of family functioning as well as actual family mealtime data provide a more objective assessment of family functioning and dietary adherence. This study also is one of only a small number of studies to focus on the experiences of families of young children with TIDM. Epidemiological reports suggest that the incidence of T1DM is increasing in children who are less than 7 years old. ${ }^{22-24}$ However, behavioural research focused on the experiences of parenting and managing T1DM in a young child is evolving at a slower rate. Therefore, this study provides important data for a previously under-represented population of families and directs research to consider the role of family functioning as a predictor of child adherence and a possible opportunity for intervention.
Clinically, the results of this study suggest that even in our present era of carbohydrate counting and fewer dietary restrictions for children with T1DM, the added burden of feeding a young child with TIDM and achieving optimal dietary adherence may disrupt family functioning. For example, current study results suggest that families who may be more disorganised at mealtimes or parents who lack the ability to manage behaviours and negative emotions (i.e. frustration) at the meal experience problems persuading their child to consume an adequate number of carbohydrates at the meal. Because patients with T1DM work to achieve normal blood glucose levels through a balance of carbohydrate intake and insulin, failure to consume the recommended number of carbohydrates in a meal could increase the risk of a hypoglycaemic episode for children. ${ }^{1}$ Moreover, if parent and child behavioural problems and poor food intake occur regularly at meals, this could promote greater stress for families of young children with T1DM. ${ }^{25}$

Thus, our results suggest that families of young children with TIDM could benefit from behavioural-based interventions that teach parents how to establish limits, set contingencies for child behaviour and eating at mealtimes, and use positive reinforcement to manage mealtime behaviours. Within the broader paediatric psychology literature, studies have demonstrated improvements in dietary adherence, child behaviour and parenting behaviours as the result of behavioural interventions targeting these strategies. ${ }^{26,27}$ These interventions could be adapted for use with families of young children with TIDM and may help to improve mealtime management and decrease family stress.

\section{Conclusion}

This is the first study linking family functioning to dietary adherence and glycaemic control in a sample of young children 
with T1DM and some limitations are noted. We elected to use observational data to assess family functioning. Observational data can provide a more objective and reliable measure of family functioning than parent report. Yet, because of the time and expense involved in collecting these data, studies may be limited with respect to sample size. Indeed, our study recruited a relatively small sample of families of young children with T1DM who were generally White and from the middle to upper middle class. Because of our relatively small sample size and the lack of heterogeneity in our sample, it is possible that results of this study are not completely generalisable. Future research is needed to examine family functioning and health outcomes in a larger sample of young children with T1DM who come from a more diverse background. Additionally, with the growing popularity of flexible insulin regimens for young children with $\mathrm{T} \mathrm{DM},{ }^{28}$ research is needed, examining family functioning in a sample of intensively managed young children to see if the relationships between family functioning and health outcomes are maintained. This study is the first to examine the relationship between family functioning at mealtimes and children's health outcomes in a sample of young children with T1DM. To examine these data, a number of correlations were run, which may increase the risk for error. We elected not to correct for the number of tests because we felt our findings were novel and lay a foundation for future research in this area. However, the reader is cautioned against over-interpreting the outcomes of this study without confirmation from future research. Finally, because this study used cross-sectional data, causality of the findings cannot be determined. Thus, it is unclear whether poorer family functioning at mealtimes results in poorer dietary adherence and glycaemic control for young children with T1DM or if dietary adherence and glycaemic control lead to greater family stress and consequently poorer functioning at mealtimes. To identify the direction of causality, research incorporating a longitudinal study design will be needed.

\section{Acknowledgements}

This research was supported in part by grants R01 DK54915 and K24 DK59973 from the National Institutes of Health/National Institute of Diabetes and Digestive and Kidney Diseases (to SWP) and grants F32 DK61121 and K23 DK076921 from the National Institutes of Health/National Institute of Diabetes and Digestive and Kidney Diseases (to SRP). This study was also supported by a post-doctoral training grant T32 DK063929 from the National Institutes of Health/National Institute of Diabetes and Digestive and Kidney Diseases (to CPW and ACM). Additional support was provided by USPHS Grant No. M01 RR 08084 from the National Center for Research Resources of the NIH. We thank the following individuals for assisting with subject recruitment, data management and manuscript preparation: Alex Castator; Molly Heidemann; Racquel Henry; Shanthi Rajan, RD; Debbie Standiford, RN; and Suzanne Summer, RD (Cincinnati Children's Hospital Medical Center). We thank Tammy DiMuzio (Cincinnati Children's Hospital Medical Center) and Scott Sapp (Animas Corporation, West Chester, PA, USA) for their assistance in obtaining blood glucose meters and testing supplies for families. We thank TheraSense, Inc for their donation of FreeStyle blood glucose meters, test strips and testing supplies. Finally, we thank the families who participated in this research project.

\section{References}

1 Silverstein J, Klingensmith G, Copeland K et al. Care of children and adolescents with type 1 Diabetes: a statement of the American Diabetes association. Diabetes Care 2005; 28: 186-212.

2 Lewin AB, Heidgerken AD, Geffken GR et al. The relation between family factors and metabolic control: the role of diabetes adherence. J. Pediatr. Psychol. 2006; 31: 174-83.

3 Miller-Johnson S, Emery RE, Marvin RS, Clarke W, Lovinger R, Martin M. Parent-child relationships and the management of insulindependent diabetes mellitus. J. Consult. Clin. Psychol. 1994; 62: 603-10.

4 Anderson BJ, Auslander WF, Jung KC, Miller JP, Santiago JV. Assessing family sharing of diabetes responsibilities. J. Pediatr. Psychol. 1990; 15: 477-92.

5 Schafer LC, McCaul KD, Glasgow RE. Supportive and nonsupportive family behaviors: relationships to adherence and metabolic control in persons with type I diabetes. Diabetes Care 1986; 9: 179-85.

6 Waller DA, Chipman JJ, Hardy BW et al. Measuring diabetes-specific family support and its relation to metabolic control. J. Am. Acad. Child Psychiatry 1986; 25: 415-18.

7 McKelvey J, Waller DA, North AJ et al. Reliability and validity of the Diabetes Family Behavior Scale (DFBS). Diabetes Educ. 1993; 19 125-32.

8 Linscheid T, Budd K, Rasnake L. Pediatric feeding disorders. In: Roberts M, ed. Handbook of Pediatric Psychology. New York: The Guilford Press, 2003; 481-98.

9 Patton SR, Dolan LM, Mitchell MJ, Byars KC, Standiford D, Powers SW. Mealtime interactions in families of pre-schoolers with type 1 diabetes. Pediatr. Diabetes 2004; 5: 190-8.

10 Stark LJ, Jelalian E, Mulvihill MM et al. Eating in preschool children with cystic fibrosis and healthy peers: behavioral analysis. Pediatrics 1995; 95: 210-5.

11 Piazza-Waggoner C, Modi AC, Powers SW, Williams LB, Dolan LM, Patton SR. Observational assessment of family functioning in families with children who have type 1 diabetes mellitus. J. Dev. Behav. Pediatr. 2008; 29: 101-5.

12 Christensen NK, Terry RD, Wyatt S, Pichert JW, Lorenz RA. Quantitative assessment of dietary adherence in patients with insulin-dependent diabetes mellitus. Diabetes Care 1983; 6: 245-50.

13 Patton SR, Dolan LM, Powers SW. Dietary adherence and associated glycemic control in families of young children with type 1 diabetes. J. Am. Diet. Assoc. 2007; 107: 46-52.

14 Hartman D. Consideration in the choice of interobserver reliability estimates. J. Appl. Behav. Anal. 1977; 10: 103-16.

15 Hayden L, Schiller M, Dickstein S et al. Levels of family assessment I: family, marital, and parent-child interaction. J. Fam. Psychol. 1998; 12 7-22.

16 Alderfer MA, Fiese BH, Gold Jl et al. Evidence-based assessment in pediatric psychology: family measures. J. Pediatr. Psychol. 2008; 33 1046-61; discussion 62-4.

17 Mitchell M, Piazza-Waggoner C, Modi A, Janicke D. Examining short-term stability of the Mealtime Interaction Coding System (MICS) J. Pediatr. Psychol. 2009; 34: 63-8.

18 Shrout PE. Measurement reliability and agreement in psychiatry. Stat. Methods Med. Res. 1998; 7: 301-17.

19 SPSS. Version 13 edn. Chicago, IL: SPSS, Inc, 2005.

20 Patton SR, Dolan LM, Powers SW. Mealtime interactions relate to dietary adherence and glycemic control in young children with type 1 diabetes. Diabetes Care 2006; 29: 1002-6. 
21 Mitchell MJ, Powers SW, Byars KC, Dickstein S, Stark LJ. Family functioning in young children with cystic fibrosis: observations of interactions at mealtime. J. Dev. Behav. Pediatr. 2004; 25: 335-46.

22 LaPorte R, Matsushima M, Chang Y. Prevalence and incidence of insulin dependent diabetes. In: Harris MI, Cowie CC, Stern PP, Boyko EJ, Reiber GE, Bennett PH, eds. Diabetes in America, 2nd edn. Bethesda, MD: National Diabetes Data Group of the National Institute of Diabetes and Digestive and Kidney Diseases, 1995; 37-45.

23 Karvonen M, Pitkaniemi J, Tuomilehto J. The onset age of type 1 diabetes in Finnish children has become younger. The Finnish Childhood Diabetes Registry Group. Diabetes Care 1999; 22: 1066-70.

24 Gardner SG, Bingley PJ, Sawtell PA, Weeks S, Gale EA. Rising incidence of insulin dependent diabetes in children aged under 5 years in the Oxford region: time trend analysis. The Bart's-Oxford Study Group. British Medical J. 1997; 315: 713-17.
25 Powers SW, Byars KC, Mitchell MJ, Patton SR, Standiford DA, Dolan LM. Parent report of mealtime behavior and parenting stress in young children with type 1 diabetes and in healthy control subjects. Diabetes Care 2002; 25: 313-18.

26 Stark LJ, Powers SW, Jelalian E, Rape RN, Miller DL. Modifying problematic mealtime interactions of children with cystic fibrosis and their parents via behavioral parent training. J. Pediatr. Psychol. 1994; 19: 751-68.

27 Stark LJ, Mulvihill MM, Powers SW et al. Behavioral intervention to improve calorie intake of children with cystic fibrosis: treatment versus wait list control. J. Pediatr. Gastroenterol. Nutr. 1996; 22: 240-53.

28 Bode BW, Sabbah HT, Gross TM, Fredrickson LP, Davidson PC. Diabetes management in the new millennium using insulin pump therapy. Diabetes Metab. Res. Rev. 2002; 18 (Suppl. 1): s14-20. 